\title{
Relationship between leadershipand employee productivity in an organization
}

\author{
${ }^{1}$ Oladipo KolapoSakiru, ${ }^{1}$ Jemilah Othman ${ }^{2}$ Aliyuyero, ${ }^{1}$ Mohammed Abdullahi, ${ }^{1}$ Narges Kia, \\ ${ }^{I}$ Department of Human Resource Development, university putra Malaysia. Malaysia. \\ ${ }^{2}$ Department of Political science, Kaduna State university, Nigeria
}

\begin{abstract}
Thispaperexaminesthe relationship thatexistsbetweenleadershipand employee productivity in an organization. Itdescribestheinterplaybetweeneffective leadership and elegance, properusage ofemployee'sabilityandmotivationeffectsinorganizationalproductivity. Leadershipoccupiesa reallysensitivepositionin almost any effective-driven modernorganization. Thispaperobservesthatamong thelabor forcein Nigeria, you will findemployeesendowedrich inabilityandwhosevaluesandcontributionshave to beutilizedand enhanceduponfor topproductivity. Itchallenges leaders inorganizationto completelyuse them incommerciallyhighproductivityandnationaleconomicdevelopment. Thepaper implicates among others, the lack ofeffectiveleadershipandefficientmanagementstylesparticularly intodayof effective-driven productioneconomy.Itunderscoresthe requirement forefficientorganizational ideas ingrained withpracticalleadershipandspiritualcapitalfor the establishmentandimplementationof organizational andsustainableproductivity among the employee in the organization.
\end{abstract}

Key word: leadership, organization, employee productivity

\section{Introduction}

Asorganizationsas well as theirconditionshavechangedrapidlyin the lastyears, a brand newtype ofleadership, one that'sless bureaucratic and much more democratic, is needed tobe able to makesurethe organization's survivaland gratification (1). It'scontendedthateffectiveleadershiphas anoptimisticeffect ontheproductivity of the organization. (2). Ultimatelyit's theperformanceof numerouspeople thatculminateswithin theproductivity of the organization, or perhaps intheachievementof organizational goals. Effectiveleadershipisinstrumentalin makingcertain organizationalperformance (3). Consequently, manyleadershipideashappen to besuggestedwithin the lasthalf centurythat arestatedto possessaffectedthe generaleffectivenessof theorganizationswherethey've beenemployed. Within thecompetitiveworldbusinessatmosphere, it is essentialthatorganizationsemployleadershipstyleswhich alloworganizationsto outliveinside adynamicatmosphere (4).

It's contended that, regardless of goals that motivated the establishment of the organization, you will find different leadership and management methods designed to offer the targets. Productivity is, generally, based on the amount and excellence of goods and services created that determines the achievement of organizational goals. Within their various researches, (5) have proven that low productivity is recorded in just about all (public) organizations in Nigeria. These studies argue how the emphases organizational managers put on achievement of organizational goals in the cost of an operating authentic leadership implanted with intellectual and spiritual capital, matched workers' interests and motivation lead hugely for this abysmal result. (6) Realizes that, in many organizations, there's insufficient cooperation between management and subordinates. This affects the contributions of additional factors of production. Every organization is really a system of interacting elements and structures that need coordination and team development. The cooperation and contributions of interacting elements ought to be elicited (7). If organizations need employees for enhanced productivity, employees also need knowledgeand practical leadership to integrate the standards of production for that enhanced productivity. Organizations therefore, need authentic and practical leadership not just to integrate the factors of production but to motivate the labor force to make sure achievement of organizational goals.

Today,contemporaryorganizationsareindicatedbysuchconstantlyalteringdynamicsasthe

complexityofpersonalization, andcompetitionneedsofpeopleinstead ofmethodsreliance upontechnologyand also theriseof theunderstandingeconomyamonga number of otherchallenges, (6). Indeed, this is actually themorereasonleadership developmentis vitaltowards thesuccessandsurvivalof theorganizationnowa lot more thanbefore. Today, themissionforresponsibleorganizational leadershipnowinvolves, education, encouragement, strengthening, andenablingthefollowerto completebetter, (6). Theessenceofleadershipinmodernorganizationsnowadaysis the fact thatleadershipshould befor theadvantage ofthefansand neveralwaysfor that enrichment from theleaders. Leadershipis all aboutdrawingpeopletogetherto obtain thetasksaccomplished, which offerthe organizationalmission. It's notaboutpositionneithercould it 
beaboutenergy. With thispositionand energycomesgreatresponsibility. The dutyto look aftersubordinatesandincrease theirsize isjust thejobof theleader. This will berelevantspecifically in an organizationwhereemployee'sbehavioris seenculturallylike apurpose ofleadershipbehavior, affectingeitherpositivelyoradverselyaround thepsycheofsubordinates, whoin reactionwithholdordemonstratesufficientbehavioralstandards (8).

\section{Leadershipand organizationalProductivity}

Therealization of businessgoalsis dependent, to somegreaterextent, onleadership. This really is explicated in(9) who posits thatorganizationalperformancenot justrelies uponthe standardofleadershipbutthatleadership, to somegreaterextent, determineshow an organization iscapable ofprogresswhen confronted withspeeding upinformationmanagementandtechnology. Leadershipsuggeststhatan individualorfactor toguide. It truly does workwithin contexts andsituations. It calls forhavingauthority, influenceandcertaincharacteristics, abilities, understanding, informationandbehaviorcharacteristics. An individualinleadershippositionfunctionsinsideasocialgroupcontextandsituations.

Heexercisesauthorityandinfluenceto be able tocorrectlylead (10). Generally, leadershipis seen asthough itwere ingrained withpersonalitygoodies. ascarceresourceincludedynamismandeffectiveness. sensitiveandproperpositioninorganizations.

An Characteristicsofleadershipthatdistinguishitlike These characteristicsexplainitsvital, justplaystherolesbutguaranteesthat workis putoncontrol effectiveleadershipnot inrecognizingthe rationally setgoalsthroughcooperativeattitudeamongoroupsand people whooperatelike asocialunit. Performanceandachievementrequirecriticalleadershipthatinspires. Managementdoesn'tachieveinisolationbutthroughmotivatedsocialgroupswithin Thesesocialgroupsact asa fantasticteamthat's organizedby asuitableandaccommodatingleader.

Effective's leader in an organization is the fact thatleadershipthat's competent, skilled, capable, innovative, competitive, motivatingandtargetoriented. It'sindicated byinnovativeand explorative management, accommodationofshadesofopinionespeciallyindividualsfrom theproper stakeholders, understandingconcerning thetechniquesandprocessforqualityitemsandservicesand knowledgeconcerning the organization's proper constituencies andcompetingvalues,(6). Organizational leadershipiscompetentenoughto becomeappliedbeing anart, ascienceso that asa method. It'smotivatingand embodied withunderstanding, valuesandvaluesofbothorganizationand also the stakeholders similarlyalong with apractical activitybasedorientedcompetencies, resourcefulness, innovativeness andmanagingcapabilities.

A superbsign ofeffective leadershipis itinvolvesaconscioustechnique ofmaking certainthe rightpeople (employees) acquiredtherequisiteunderstandingin theproper time. Itguaranteesthe employeesorindividuals areaidedto gain access to, shareand setinformationintoactionin a waythe organizationeffectivelyrealizesitsgoalsthroughperformance (11). It'sfueledandpowered byan unflagging energyandenthusiasmto make sure thatworkers arecompetent, knowledgeableandmotivatedto attainperformance. Thisabouteffective's leaders $\quad$ isexpedienttakinginto cognizance globalcompetitiveanddynamiceconomicatmosphereis understanding-driven. An effective leader therefore, remains themost crucialproperresourcethat'srequisitelike aneffectiveengineformotivationofemployeesas well as forproduction. Leadership involvesa continuingprocedure forunderstandingcreationandapplicationthroughaccess, documentationanddistribution. Zinc heightens organization's competitivestrategyalong with its effectives (12). In aclearcaseofcontingencysituation, thesocialrelationsbetween yourleaderand thesubordinatesexposethe amount ofconfidence, mutualtrustandrespectwhichsubordinatesdress intheirleader. Wherethesepositivefitsexist, thesubordinatesaremotivatedtogreaterproductivity. The characterandtaskstructurefrom thejobassignment goal lengthymethod to predict the quality ofclearnessandreliance uponstipulatedmethods. Additionally, itdescribeshowknowledgeablethe leadershipis. Howstructuredor unstructured an activityorpleasure isand it isinclination towardstechnologicalchangedetermineshowquickdecisiontakingis going to belike. Leadership styles in an organization determine the employee's productivity in that organization (5).

\section{Data and discussion}

\section{Methodology}

Thesettingcharacteristicsof theparticipantswithin thethree Ministries ofworks, education, andhealth in Nigeria, $\quad$ indicatethatabout $\quad 56 \%$ arewomenwhile $44 \% \quad$ aremales. Thejuniorstaffonannualsalaryongradelevelsevenlower $(<\mathrm{GL} .7)$ in thesampleareabout $60 \%$ whilestaffongradeleveleightandabove (>GL.8andabove) areabout $40 \%$. Lots ofcivil servants (53\%) arewithin theage rangesof 31-50years, showingthatbyage, they have gotmoreyearsfor them to spend withthe government. The educationalqualificationsindicatethat $55 \%$ retain firstdegreewhile $25 \%$ haveDiplomaandGreaterNationalDiplomaCertificates. $20 \%$ haveSeniorSchoolCertificate. Amongpeople that 
havefromfirstdegreeandabove, basicallya small $5 \%$ in theservingstaffgottheirlevelsbyselfsponsorshiporbackedgovernment in-service programmesorthroughleaveofabsence. It'sunhealthyeffectsongovernmentrecommendations onhumancapitaldevelopment, utilization ofinformationandunderstandingandmissionforenhancedproductivityin this particularcompetitiveand $\begin{array}{llll}\text { understanding-driven } & \text { economyandproduction. } & \text { Only } & 3 \%\end{array}$ peopleinmanagementpositionhaveattendedmanagementtrainingprogrammesorcoursesin the lasttwo years. This can becreditedto non-ease ofaccess tofundsand government's nonchalantattitudetowardsmanagementtrainingandbusinessdevelopmentgenerally. If thisinvolvesyearsinside theserviceofpresidency, the truth is $47 \%$ in theparticipantscome inservicebetween 11 and $20 \mathrm{yrs}, 23 \%$ haveofferedbetween6and10 years, 20\% come inservicefrom 21 to35years. Only10\% haveofferedthan5 years. Similarly, it appears, within thedata, that numerousmoreemployees $(80 \%)$ willassistthe governmentlonger. Alternatively, when thepercentageisdeclinedutilization ofinformation, newideasandknowledge, as a result ofinadequatetrainingprogrammesandpoorhumancapitaldevelopment, thenservicedeliveryandproductivitywill remainatlower ebbs in Nigeria.

\section{Objectives 1: To examine the relevance of effective's leadership in the organization}

One of theobjectivesfrom theanalysisis always tounderscoretherelevanceofeffective'sleadershipinthe organizations. Effectives leaderspossesses analluringroleof creatingcertainasustainabletechnique ofcreativity, being able toview, disbursing, storingand usingknowledge andinformationinside theorganization. Inconsidering the relevance from theallpurposefulcatalystinorganization, thesestudieswas carried out with 200 participants from the three ministries mention in this paper, and it was observedthat $30 \%$ in theparticipants averred thateffectivesleadershipisindispensableforbeginningand usingorganizationalinitiativesand techniques, making sureleadership followership relationship (20.5\%) andintegratingpropergroupsin addition to constituencies inorganization (22.5\%). About $27 \%$ in theparticipantsestablishedthat therequirement ofeffectivesleadershipinorganizationis always toidentifyandmotivatehighabilityemployeesto have theability toassignthemresponsibilitiesto find the bestproductivity

Table 1

\begin{tabular}{|c|c|c|c|}
\hline & Relevant variables & Frequency & Percentage \\
\hline A & $\begin{array}{l}\text { Initiating and } \\
\text { implementing } \\
\text { organizational strategies }\end{array}$ & 60 & 30 \\
\hline B & $\begin{array}{l}\text { Integrating strategic } \\
\text { groups in organization }\end{array}$ & 41 & 20.5 \\
\hline $\mathrm{C}$ & $\begin{array}{l}\text { Identifying } \\
\text { motivating high } \\
\text { workers }\end{array}$ & 45 & 22.5 \\
\hline \multirow[t]{2}{*}{$\mathrm{D}$} & $\begin{array}{ll}\text { Ensuring } & \text { leadership } \\
\text { followership relationship }\end{array}$ & 54 & 27 \\
\hline & Total & 200 & 100.0 \\
\hline
\end{tabular}

Thisfindingindicates thatfrom the result that, about $73 \%$ in theparticipantsagreedthe indispensability of effectivesleadershipis dependant onitsroleof articulating the companyinitiativesand techniques, organizingandmatchingthe variousproper constituencies andinterestgroups (stakeholders) inside theorganizationandidentifyingandmotivatingthe topabilityemployees, allfor thegreaterproductivityin theorganization. Essentially, itaimstoaggregatetheinitiativesand techniques, thecollectiveunderstandingandculturein theorganization, informationandpracticesin theorganizationandmakesevery one of theseavailable toindividualemployeesandbusiness workgroups for use. This tilts towardsmotivatingthe workforcein waystheirefforts, knowledge and abilitiesbecomecollectiveandorganizationassetsforproductivity. Thisfindingauthenticates (13) and (5) studiesthatlooked fortorelatethe value ofknowledge,effective'sleadershipand inspiringinstrumentsfororganizationalproductivity. Thiscertifies, (6)positionthateffective'sleadershipencourages collaborative effortandopennessamongproperconditionsinorganizationforsuccessiveimplementationof economicinitiatives.

Objectives 2: Identifying leadership characteristics among the leader in the organization 


\begin{tabular}{|l|l|l|l|}
\hline & Characteristics & Frequency & Percentage \\
\hline a & $\begin{array}{l}\text { My boss is competent, } \\
\text { competitive and skillful }\end{array}$ & $\mathbf{2 4}$ & $\mathbf{1 2}$ \\
\hline b & $\begin{array}{l}\text { My boss is all-knowing } \\
\text { and emphasizes authority } \\
\text { structure }\end{array}$ & $\mathbf{5 2}$ & $\mathbf{2 6}$ \\
\hline c & $\begin{array}{l}\text { My boss is information } \\
\text { conscious and flexible }\end{array}$ & $\mathbf{1 6}$ & $\mathbf{8}$ \\
\hline d & $\begin{array}{l}\text { My boss emphasizes } \\
\text { rigidity of rules }\end{array}$ & $\mathbf{5 5}$ & $\mathbf{2 7 . 5}$ \\
\hline e & $\begin{array}{l}\text { My boss encourages } \\
\text { creativity, independence } \\
\text { and group decision } \\
\text { making }\end{array}$ & $\mathbf{2 7}$ & $\mathbf{1 3 . 5}$ \\
\hline f & $\begin{array}{l}\text { My boss enjoys mutuality } \\
\text { and cooperative workshop }\end{array}$ & $\mathbf{2 6}$ & $\mathbf{1 0 0 . 0}$ \\
\hline
\end{tabular}

Intrying torecognizeifleader inthe organizations, especiallygovernmentinstitutions, possessthe characteristicsofeffectives leadership, Table2signifies that $26 \%$ in theparticipantsseetheirbossesasallknowing, emphasizingauthorityrules (27.5\%). Only13.5\% see thebossesencouragecreativity, independenceandgroup decision-making. About $12 \%$ recognizedtheirbossesarecompetentandskillful, informationconsciousandversatile (8\%) and enjoin mutuality andcooperative workgroup (13\%). Thisfindingsignifiesnot only aclosed organizational ulturemanifestation ofinformation hording butan organizationwherescientificmanagementpractice, authoritybasedtraditions, commandandcontrolmanagementandleadershippracticesare normal. This can beunacceptablein this particularmillenniumthatadvocates decentralization of decision-making, informationtalking aboutand participatory management (5). This can bemanifestation ofgovernment ministries andinstitutionswhereauthoritybasedtraditionremainsgreatlyvalued.

This finding indicates that there are no enough effective's leadership characteristics among the leaders in the organization under study.

Objectives 3: To examine the relationship between leadership, employee motivation and productivity

This objective sought to examine the relationship between leadership, workermotivationandproductivity. Table3shows thateffective'sleadershipencouragesamong others: utilization ofnewideasandinformation (27.5\%), practical activity-based competence (31.5\%) andprogression ofabilitiesandleadershiptraining (28.5\%).

\begin{tabular}{|c|c|c|c|}
\hline & Variables & Frequency & Percentage \\
\hline $\mathrm{a}$ & $\begin{array}{l}\text { Effectives leadership } \\
\text { encourages access to new } \\
\text { ideas and information }\end{array}$ & 55 & 27.5 \\
\hline b & $\begin{array}{lr}\text { Effectives } & \text { leadership } \\
\text { encourages } & \text { development of } \\
\text { capacities and leadership } \\
\text { training }\end{array}$ & 57 & 28.5 \\
\hline $\mathrm{c}$ & $\begin{array}{lr}\begin{array}{l}\text { Effectives } \\
\text { encourages }\end{array} & \text { leadership } \\
\text { alone } & \text { productivity }\end{array}$ & 10 & 5 \\
\hline $\mathrm{d}$ & $\begin{array}{l}\text { Effectives leadership } \\
\text { encourages practical activity } \\
\text { - based competence }\end{array}$ & 63 & 31.5 \\
\hline $\mathrm{e}$ & $\begin{array}{l}\text { Effectives leadership is } \\
\begin{array}{l}\text { domineering and non- } \\
\text { productive }\end{array} \\
\text { Total }\end{array}$ & 200 & 100.0 \\
\hline
\end{tabular}


Thesefactorsare adjudged

beingsubstantiallyrelated

Aworkerthatcravesforaccessforinformationandunderstandingneeds to

withmotivation. toworkouthiscapabilitiesindividually (withminimumsupervision). throughcapacitydevelopmentprogrammesandtraining, being explorative, articulateand innovatively creative. He'sratheraresourceto him selves, the organizationas well as thenationgenerally. Just like amotivatedworker, heidentifiesand commits hisabilitiesand abilitiestoproductivitytherefore, theachievementoforganizationsetobjectives.

\section{Conclusion}

Effectives leadershipremainsrecognized in the organization, in this particularanalysisasthatleadershipthat'scompetent, skilled, innovative, competitive, target-oriented capable tomotivatingorganizations 'work forcetowardsgreaterproductivitythroughappropriatecoordinationin theproper constituencies inside theorganization. It isnota specialistbasedtraditionalleadershipthathordesknowledgeand information or upholds commandandcontrol"managementandleadershiporientation, It is thetypethat'sappropriatetocoordinatebothhumanandmaterialassetsinorganizationfor productivity in the effective driven productioneconomy.

The studyobserved, among others, the possible lack ofeffectiveleadershipinside the Nigerian economyand therefore re-iterates the requirement toestablishsameto boostworkermorale, coordinatethe nation's assetsandimproveproductivity. Itrecognizedthestandards militating again extablishentofeffective's leadership in an organization in Nigeria. Itarguesthat therolesofgovernmentinimprovingorganizational productivity includes encouragement of humancapacitybuildingandworkergettingpracticalleadershipandspiritualcapital-a selflessly divine-given existence pressurethatencouragesan individualtowardsgoodandmaking sureexcellencewithoutaffixingpricesto one's efforts.

\section{References}

[1]. Maley, D. 1995. Leadership and mobilizing potentia, Human Resource Management, 10(1):8-16.

[2]. Charlton (2000). A Study to improve Organizational Performance: A View from SHRM. Journal American Academic Business, 4: 289-294.

[3]. HellriegletB. (2004) Organization Theory: Structure, Decision and Application. New Jessy: Prentice-Hall International Inc.

[4]. Bass, B.M. (1997) Does the transactional-transformational leadership paradigm transcend organizational and national boundaries? American Psychologist, 52: 130-139.

[5]. Iheriohanma, E.B.J. (2006) Perceiving Worker's Interest in Participatory Management: Issues and Challenges. International Journal of Social Sciences, 5, $113-129$.

[6]. Mbogu, G. C. (2001)_Implications of Leadership Styles of Administrators on Job Performance in Two Selected Organizations in Owerri, Imo State' Unpublished Thesis. Calabar: University of Calabar . Nigerian Institute of Social and Economic Research (NISER) (1998) _Capacity Building Indicators in Public and Private Sectors'. NISER Annual Monitoring Research Project (NAMRP). Ibadan.

[7]. Akunyili, D.N. (2008) The Contemporary Nigerian Organization and its Leadership Challenges for Women Managers. Management in Nigeria: Journal of Nigerian Institute of Management, 44 (2), 14-21.

[8]. Iheriohanma, E.B.J. (2002) Contemporary Issues in Organization Theory and Management . 2nd edition. Owerri: CREMD Publishers.

[9]. Ezuluke R.E. (2001)_An Appraisal of Manpower Development Practice in Agricultural Institution: A Case Study“. Benin Journal of Social Sciences, 2 (2), $99-109$.

[10]. Boisot, M.H. (1998) Knowledge Assets. New York : Oxford University Press.

[11]. Blackler, F. (1995) Knowledge, Knowledge Work and Organizations: An Overview and Interpretation. Organization Studies, 16 (6), 1021-1046

[12]. Reber, A. S. (1995) The Penguin Dictionary of Psychology. Second Edition, London: Penguin books.

[13]. Iheriohanma, E.B.J. (1997) Motivating Organization's Workforce: A Project Management Instrument Against Labour Turn-over. Journal of Project Management Technology, 1 (1), 56-65 